\title{
Trends in practice and safety measures of epidural analgesia: report of a national survey
}

\author{
Elke M.E. Bos ${ }^{1}$, Maartje E. Schut ${ }^{1}$, Marcel de Quelerij ${ }^{2}$, Cor J. Kalkman³, Markus W. Hollmann' ${ }^{3}$, Philipp Lirk
}

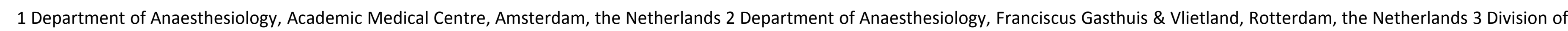

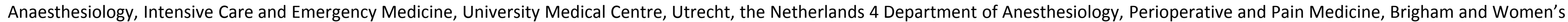
Hospital, Harvard Medical School, Boston, MA, USA

\section{Introduction}

The clinical use of epidural analgesia has changed over past decades. Minimally invasive surgery and emergence of alternative analgesic techniques have led to a decline in its use. Additionally, there is increasing awareness of the patient-specific risks for complications such as spinal haematoma and abscess. Local guidelines for management of these severe neurological complications during or after epidural analgesia, i.e. 'epidural alert systems', have been introduced in some hospitals to coordinate and potentially streamline early diagnosis and treatment. How widely such protocols have been implemented in daily practice is unknown.

\section{Methods}

We conducted a web-based survey in order to analyse trends in practice, key indications, safety measures, reporting and management of complications of epidural analgesia in the Netherlands. In January 2016 a questionnaire was sent to all hospitals in the Netherlands. Last questionnaires were collected in August 2016. Children's hospitals, private practices and outpatient clinics were excluded. For each location one anaesthesiologist (typically the head of service or clinical lead for regional anaesthesia services) was asked to fill in the questionnaire or identify the most appropriate anaesthesiologist to answer the questionnaire based on clinical involvement in neuraxial anaesthesia. We used descriptive statistics to analyse data.

\section{Results}

The questionnaire was sent to 131 individual locations; 37 of these locations were excluded since no epidural procedures were performed at their site (35 hospitals) or the hospital performed epidural analgesia solely in children ( 2 hospitals). Ultimately, 94 locations were considered eligible for analysis. Eighty-five of 94 questionnaires $(90 \%)$ were returned for analysis. Key indications for epidural analgesia are thoracotomy, laparotomy of the upper abdomen and abdominal cancer debulking, see Table 1 .

Regarding trends in epidural analgesia use, 55\% reported a trend towards decreased use of perioperative epidural analgesia, while $68 \%$ reported increasing use of epidural analgesia for labour, see Figure 1. Nineteen percent (16 hospitals) could report exact numbers of perioperative epidural procedures performed per year, suggesting a detailed registration of epidural procedures, $32 \%$ reported accurate estimates (expected deviation from the true number less than $10 \%$ ) and $49 \%$ could only provide rough estimates. For labour epidural procedures these numbers were $38 \%$ ( 26 hospitals), $35 \%$ and $27 \%$ of hospitals, respectively. In case of spinal haematoma after epidural analgesia the respondent hospitals reported diverse management strategies. Management was determined ad-hoc/no preagreed management strategy was present in $46 \%$ of hospitals for patients with spinal haematoma presenting with pain and neurological deficit, see Figure 2 .

\begin{tabular}{|c|c|c|c|c|c|c|c|c|c|c|}
\hline & $\begin{array}{l}\text { EA- } \\
\text { First } \\
\text { choice }\end{array}$ & $\begin{array}{l}\text { EA- } \\
\text { Individual } \\
\text { patient }\end{array}$ & $\begin{array}{l}\text { EA- } \\
\text { (Almost) } \\
\text { never }\end{array}$ & $\begin{array}{l}\text { Preferably } \\
\text { PVB }\end{array}$ & $\begin{array}{l}\text { Dependent } \\
\text { on size of } \\
\text { incision }\end{array}$ & $\begin{array}{l}\text { Preferably } \\
\text { abdominal } \\
\text { wall block }\end{array}$ & $\begin{array}{l}\text { Preferably } \\
\text { SA }\end{array}$ & $\begin{array}{l}\text { Preferably } \\
\text { PNB }\end{array}$ & $\begin{array}{l}\text { Preferably } \\
\text { remifentanil } \\
\text { IV-PCA }\end{array}$ & Other \\
\hline Thoracotomy & $82 \%$ & $10 \%$ & $4 \%$ & $1 \%$ & - & - & - & - & - & $4 \%$ \\
\hline Thoracoscopy & $33 \%$ & $45 \%$ & $19 \%$ & $2 \%$ & - & - & - & - & - & $1 \%$ \\
\hline Mastectomy & $2 \%$ & $8 \%$ & $77 \%$ & $13 \%$ & - & - & - & - & - & $0 \%$ \\
\hline Laparotomy up. abdomen & $80 \%$ & $12 \%$ & $0 \%$ & - & $4 \%$ & - & - & - & - & $5 \%$ \\
\hline Laparotomy low. abdomen & $32 \%$ & $24 \%$ & $7 \%$ & - & $32 \%$ & $1 \%$ & - & - & - & $5 \%$ \\
\hline Laparoscopy up. abdomen & $12 \%$ & $31 \%$ & $55 \%$ & - & - & - & - & - & - & $2 \%$ \\
\hline Laparoscopy low. abdomen & $4 \%$ & $24 \%$ & $73 \%$ & - & - & - & - & - & - & $0 \%$ \\
\hline Abdominal debulking & $77 \%$ & $18 \%$ & $2 \%$ & - & - & - & - & - & - & $4 \%$ \\
\hline Total hip prosthesis & $0 \%$ & $0 \%$ & $29 \%$ & - & - & - & $59 \%$ & $4 \%$ & - & $8 \%$ \\
\hline Knee prosthesis & $2 \%$ & $1 \%$ & $22 \%$ & - & - & - & $52 \%$ & $9 \%$ & - & $13 \%$ \\
\hline Obstetrics & $46 \%$ & $39 \%$ & $6 \%$ & - & - & - & - & - & $1 \%$ & $8 \%$ \\
\hline
\end{tabular}

\section{Epidural Alert System}

As a potential safety measure, a local protocol for the diagnostic workup and management of spinal complications of epidural analgesia, i.e. an 'epidural alert system', was implemented in $45 \%$ of hospitals $(n=38)$. The diagnostic workup would be coordinated by the treating specialist (e.g., orthopaedics, surgery, etc.) in 3 hospitals (8\%), by the consulted neurologist or neurosurgeon in one hospital $(3 \%)$, by the anaesthetist on call in 28 hospitals (74\%) and by an Acute Pain Service in 4 hospitals (11\%). In 2 hospitals (5\%) with an epidural alert protocol it was not clear which clinician is responsible for the coordination of the protocol.

Table 3 Indications for epidural analgesia. EA: epidural analgesia, PVB: paravertebral block, SA: spinal anaesthesia, PNB: peripheral nerve block, IV-PCA: intravenous patient controlled analgesia, up: upper, low: lower. Key to symbols: - : No answeroption in this question, Other : Multiple answer-options given
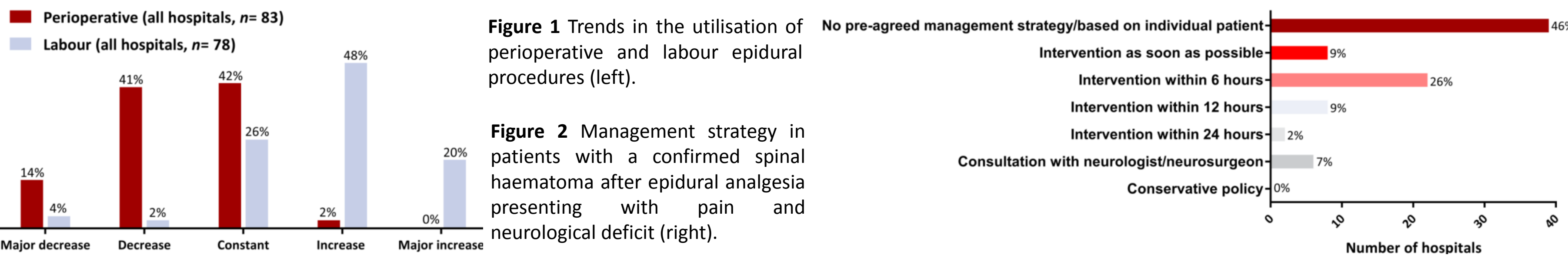

Discussion \& Conclusion

Anaesthesiology departments in the Netherlands appear to follow the worldwide trend of decreased use of perioperative epidural analgesia. Most hospitals in the Netherlands do not have a pre-agreed protocol for the diagnostic workup and management of severe neurological complications of epidural analgesia. A possible limitation of this survey is that some questions may be answered or interpreted ambiguously between respondents. Also, one anaesthetist per hospital completed the questionnaire. In reality, it is plausible that differences of opinion exist within an anaesthesiology department. Conversely, a strength of our survey is the high response rate of $90 \%$. Therefore this survey adequately reflects the current practice and safety of epidural analgesia practice in the Netherlands. In order to streamline diagnostic procedures and thereby accelerating potential interventional therapy for rare complications of epidural analgesia such as spinal haematoma or abscess, we consider the implementation of local Epidural Alert Systems of importance. 